\title{
Avaliação de usabilidade e de experiência de jogo digital educacional: uma experiência com o SUSCity.
}

\author{
Ivana Lima - PPGIE | UFRGS - ivanaufrgs@ gmail.com \\ Denise Bueno - Faculdade de Farmácia - denise.bueno@ufrgs.br \\ Gabriela Trindade Perry - Departamento de Design e Expressão Gráfica | UFRGS - \\ gabriela.perry@ufrgs.br
}

\section{Resumo}

Neste artigo relata-se a avaliação do jogo digital educacional SUSCity, elaborada como material de apoio para a disciplina de Saúde Coletiva e Bioética, do curso de Farmácia da UFRGS. Esta avaliação foi realizada na metade do semestre letivo de 2016/02, por 46 estudantes do primeiro semestre. Antes de utilizar o jogo, os estudantes haviam tido uma aula presencial sobre o assunto do jogo. A avaliação foi feita sob a forma de questionários, aplicados após o uso do jogo, e focou dois aspectos: objetivos e de experiência (de fluxo). Os resultados evidenciaram que os estudantes não atingiram o estado de fluxo durante o jogo. Uma avaliação de consistência indica que os dados do questionário sobre aspectos objetivos não possuem confiabilidade, e portanto não podem ser usados para concluir sobre o jogo.

Palavras chaves: Avaliação de jogos educacionais; Usabilidade de Jogos educacionais; Jogo digital educacional; Experiência de fluxo.

\begin{abstract}
In this paper we report the evaluation of the educational digital game SUSCity, elaborated as support material for the discipline of Collective Health and Bioethics, of the Pharmacy course at UFRGS. This evaluation was carried out in the middle of 2016/02, by 46 junior students. Before using the game, the students had a lecture on the subject of the game. The evaluation was done with questionnaires, applied after playing the game, and focused on two aspects: objectives and experience (flow). The results showed that the students did not reach the flow state during the play. A consistency evaluation indicated that the questionnaire data on objective usability aspects is not reliable, and therefore cannot be used to conclude about the game.
\end{abstract}

Key words: Educational game evaluation; Educational game usability; Educational digital game; Flow experience.

\section{Introdução}

A popularidade dos jogos digitais em contextos educacionais tem aumentado. Contribuíram positivamente para este cenário os constantes avanços de software e hardware, que têm facilitado o desenvolvimento de jogos com alta qualidade, mesmo com equipes reduzidas e com baixo orçamento. Além disso, os rápidos avanços das tecnologias de comunicação e as melhorias da capacidade de transmissão de dados estão possibilitando a expansão do púbico consumidor de jogos digitais, o que abre inúmeras possibilidades de jogos para serem exploradas como ferramenta no contexto educacional.

O grande potencial dos jogos como ferramenta de ensino talvez seja derivado da possibilidade de eles serem espaços de resolução de problemas altamente envolventes (GEE, 2008; GEE, 2009). Os jogos fariam com que os jogadores pensem como cientistas, pois possuem um ciclo típico da ciência experimental: "postular hipóteses, 
explorar o mundo, obter uma reação, refletir sobre os resultados, reexplorar para obter melhores resultados" (GEE e MORGRIDGE, 2007). No entanto, ao mesmo tempo em que parece evidente que os jogos têm o potencial para funcionar como ferramentas pedagógicas valiosas, há pouca evidência de que eles produzem resultados educacionais duradouros, confiáveis, válidos e longos, ou que jogos são mais eficientes do que as estruturas tradicionais de ensino (O'NEIL e WAINESS, 2005; LINEHAN e KIRMAN, 2011; BROM et al, 2011). O'Neil e Wainess (2005) analisaram 19 trabalhos, de 1990 a 2005. O objetivo era verificar a hipótese que resultados positivos podem ser atribuídos ao design instrucional, não aos jogos per se. Esta hipótese está em consonância com a hipótese de Richard Clark - segundo a qual o que importa para o desempenho de aprendizagem é o método de ensino, e não a mídia (CLARK, 1994; KOZMA, 1994; CLARK e FELDON, 2005). Li e Tsai (2013) também apresentaram um artigo de revisão de 31 estudos que descrevem resultados de jogos educacionais. Uma conclusão importante deste estudo é que "embora os fundamentos teóricos aplicados [nesses estudos] tenham variados desde as mais amplas perspectivas de aprendizagem até princípios mais específicos, a maioria destes estudos (14) aplicou tais teorias [nos jogos] a um nível muito superficial" (p. 885). Os autores concluem que os jogos descritos nos artigos analisados não aproveitaram plenamente o potencial de gêneros (tipos de jogo) e plataformas, sendo focados principalmente na resolução de problemas ou memorização de conceitos (p.889).

Por este motivo, direcionamos nossos esforços para a criação e a avaliação de um jogo educacional cujo contexto é o tratamento de doenças por meio do SUS Sistema Único de Saúde. O objetivo dessa pesquisa é apresentar a avaliação de aspectos da usabilidade e da experiência do usuário.

\section{Avaliação de usabilidade e de experiência do usuário}

A Avaliação de Usabilidade (uma subárea da Interação Homem Computador) em sistemas digitais é um assunto de reconhecida importância, e vem sendo investigada há bastante tempo. Provavelmente os maiores desafios impostos da área são relacionados ao seu caráter multidisciplinar e à dificuldade de medir construtos que são difíceis de definir. Reconhecendo esta complexidade, a ISO 9241-11 separa a avaliação de usabilidade de aspectos subjetivos dos objetivos, definindo-a como a medida pela qual um produto pode ser "usado por usuários específicos para alcançar objetivos específicos com efetividade, eficiência e satisfação em um contexto de uso específico". As variáveis de interesse da medição relacionadas à satisfação podem ser categorizadas como "de experiência do usuário", ou seja, de fundo subjetivo. Bevan (2009) afirma que apesar de avaliações de eficiência e eficácia terem perfil objetivo, enquanto satisfação tem um perfil subjetivo, não há diferenças entre os instrumentos e métodos de avaliações.

Ter boa usabilidade é uma característica fundamental para qualquer artefato digital ou não - e proporcionar uma boa experiência também é altamente desejável. No entanto, historicamente o foco recaiu com mais ênfase na avaliação de aspectos objetivos da usabilidade (eficiência e eficácia) por uma questão de perfil da tecnologia, pois o uso da tecnologia digital era um meio (de trabalho) e não um fim (de lazer). Com o desenvolvimento tecnológico, tanto de software como de hardware, a preocupação com a experiência acabou ocupando um lugar fundamental na avaliação de um artefato digital, culminando no crescimento da área de Experiência do Usuário. Para demonstrar a importância da componente da experiência, Hassenzahl et al. (2000) propôs um modelo que relaciona aspectos hedônicos e ergonômicos, sendo que cada um destes aspectos tem uma participação de $50 \%$ na construção da percepção do apelo de um produto. Ele ainda verificou que as qualidades hedônicas e ergonômicas de um artefato 
são percebidas distintamente, o que confirma a adequação da definição de usabilidade da ISO 9241-11.

Todavia, a avaliação de aspectos subjetivos tem sutilezas difíceis de contornar, o que trouxe a avaliação de usabilidade de volta ao centro das atenções da área acadêmica de Interação Homem Computador. Estas sutilezas são derivadas da natureza composta das nossas emoções, humores e afetos (tudo aquilo que influencia o que Hassenzahl chama de "aspectos hedônicos"). Segundo Quigley et al (2014), afeto é uma referência a sentimentos difusos, que não se consegue explicar o que está sentindo. Por outro lado, emoção refere-se a sentimentos que são uma resposta a um evento específico, e são estados discretos e fáceis de identificar, como raiva, medo, tristeza, desgosto e felicidade. Em ambos os casos, a complexidade da mensuração relaciona-se com a forma como se conceitua afeto. Segundo Boehner et al (2007), "emoções são fundamentadas culturalmente, experienciadas dinamicamente e, em alguma medida, construídas na interação". Isso implica que as componentes das emoções (e do afeto) não são separáveis, e que, portanto não podem ser objetivamente acessadas. Boehner et al (2007) afirmam que nossas teorias sobre cognição e emoções não as consideram como fenômenos construídos durante a interação, e sim como estados mentais porque é esta a explicação coerente com o modelo científico que usamos. Além disso, para mensurar emoções e afetos é preciso considerá-los como resultado da interação entre componentes, o que torna a visão teórica difícil de realizar.

\subsection{Avaliação de usabilidade e da experiência do usuário, em jogos educacionais}

Quando o assunto são jogos digitais, a importância relativa de aspectos afetivos pode ser ainda maior. E se considerarmos que a indústria dos jogos é a indústria de entretenimento com maior faturamento, compreender como realizar avaliações destes fatores se torna fundamental. Considerando o recorte dos jogos digitais educacionais, adiciona-se às variações de interesse a interação entre o ato de jogar e a aprendizagem, e a complexidade deste problema deu origem à uma vasta produção científica, que se espalha pelos campos da Educação, Interação Homem Computador e Psicologia. No caso deste artigo, o recorte envolve a avaliação de aspectos objetivos e de fluxo, um estado afetivo que parece estar relacionado com o incremento no desempenho dos estudantes.

Em se tratando de softwares para uso profissional (como editores de texto, de planilhas, de vídeo, de gestão), a avaliação de aspectos objetivos (eficácia e satisfação) teve precedência sobre a avaliação da experiência. E com jogos ocorreu o mesmo priorizou-se a compreensão das mecânicas e dos elementos da interface. Contudo, logo a maior parte das pesquisas relacionadas à avaliação de jogos (educacionais ou não) voltou-se aos aspectos afetivos. Talvez o exemplo mais antigo seja o de Malone (1982), que buscou compreender o que torna os jogos tão divertidos, e a partir disso, criou uma lista de heurística para o design de jogos. Mais recentemente, Pinelle et al (2008) apresentaram um modelo amplo para avaliação de jogos, também através de heurísticas. Desurvire e Wiberg (2009) também desenvolveram um conjunto de heurísticas para avaliação de jogos, e chamaram-no de PLAY (Game Playability Principles), com foco em jogos do tipo Real-Time Strategy (RTS), Action Adventure e First-Person Shooters (FPS). Este conjunto foi baseado em um trabalho anterior dos mesmos autores, também voltado à avaliação de jogabilidade, chamado HEP (Heuristics to Evaluate Playability), voltados ao gameplay, usabilidade, mecânica e enredo (DESURVIRE e WIBERG, 2008). Ainda em relação à ferramentas voltadas à avaliação de jogos por especialistas, Kiili et al (2014) elaboraram um framework onde se pode avaliar a capacidade de um jogo educacional incentivar a experiência de fluxo. Considera-se que este tipo de 
ferramentas é muito útil durante a etapa de projeto (ou design) de jogos, já que, por terem uma natureza qualitativa, permitem que sejam explorados diferentes soluções para os problemas detectados. Porém, como Stanton e Young (1999) alertam, é preciso que o avaliador (o sujeito que utiliza a ferramenta) seja um especialista tanto no domínio (o jogo) como na ferramenta de avaliação (o conjunto de heurísticas ou o framework).

Ferramentas de aplicação mais simples, cujos resultados possam ser expressos quantitativamente, permitindo comparações e correlações (entre jogos, por exemplo) também começaram a atrair a atenção de pesquisadores da área de avaliação de experiência. Assim, diversas escalas e questionários podem ser encontrados na literatura, voltados à medição de construtos bastante específicos. Por exemplo, Gonzalez e Vela (2014), também usaram o conceito de jogabilidade para medir a qualidade da experiência com o jogo, porém o fizeram através de um instrumento baseado nos seguintes construtos: intrínseco (relacionado à mecânica, regras e níveis); mecânico (relacionado à operação do sistema e seus bugs); interação (elementos de interface); interpessoal (em jogos de mais um jogador). Além disso, usaram uma variável fisiológica não invasiva (frequência cardíaca) para triangular com os resultados da escala desenvolvida.

\subsection{Avaliação da experiência de Fluxo}

Fluxo é um estado mental caracterizado pela atenção concentrada e pela intensa satisfação durante a realização de uma tarefa intrinsicamente interessante. Esta experiência pode ser intensificada por características pessoais - como auto regulação, segundo Chen e Sun (2016) - e propriedades da tarefa, sendo a mais significativa a habilidade demandada do sujeito para que ela seja realizada. Este balanço entre habilidade e dificuldade é o que faz com que o jogador entre no estado de fluxo. $\mathrm{O}$ balanço entre a habilidade e a dificuldade é sutil, e pode facilmente provocar experiências negativas como ansiedade e tédio, como mostra o Quadro 1. O estado de fluxo pode ser reconhecido como imersão e engajamento.

Quadro 1. Balanço entre habilidade e desafio, relacionado à experiência de fluxo.

\begin{tabular}{ll|l|l}
\multicolumn{3}{c}{ HABILIDADE } \\
\cline { 2 - 4 } Muita exigência & Pouca exigência \\
\cline { 2 - 4 } \\
\cline { 2 - 4 } & Muita exigência & Fluxo & Ansiedade \\
\cline { 2 - 4 } & Pouca exigência & Relaxamento & Apatia
\end{tabular}

Hamari et al. (2016) investigaram a relação entre fluxo e aprendizagem, avaliando como os componentes do fluxo se relacionavam com o desempenho dos estudantes que participaram da pesquisa. Os autores encontraram uma correlação positiva entre engajamento e aprendizagem, porém não entre imersão e aprendizagem. Desafio foi um bom preditor de aprendizagem, ao passo que habilidade não. Por este motivo, acreditamos que avaliar fluxo seria importante em relação à qualidade de jogos educacionais.

\subsubsection{O questionário EduFlow}

Partindo de estudos sobre quatro dimensões do fluxo (absorção cognitiva; concentração e imersão na tarefa; alteração da percepção do tempo; perda da auto consciência; (iv) bem-estar), Heutte et al (2014) propuseram a escala Eduflow, instrumento que foi desenhado para ser utilizado em ambientes de aprendizagem. Por 
este motivo, escolhemos este instrumento para avaliar a experiência de fluxo. O Quadro 2 lista os enunciados das questões, associadas ao construto que elas avaliam.

\begin{tabular}{l|l}
$\begin{array}{l}\text { Quadro 2 - Construtos e enunciados do EduFlow. } \\
\text { Construto }\end{array}$ & Enunciado \\
\hline Absorção cognitiva & $\begin{array}{l}\text { Questão 01. Me senti capaz de atender às exigências da situação } \\
\text { Questão 05. Eu sinto que o que eu faço está sob meu controle. } \\
\text { Questão 09. Sei o que tenho que fazer em cada etapa da tarefa. }\end{array}$ \\
\hline $\begin{array}{l}\text { Alteração da percepção } \\
\text { do tempo }\end{array}$ & $\begin{array}{l}\text { Questão 02. Não notei a passagem do tempo. } \\
\text { Questão 06. Eu sinto que o tempo está voando muito rápido. } \\
\text { Questão 10.O tempo parece fluir através de uma forma diferente do que } \\
\text { nunca. }\end{array}$ \\
\hline $\begin{array}{l}\text { Perda } \\
\text { autoconsciência }\end{array}$ & $\begin{array}{l}\text { Questão 03. Não me preocupo com o que os outros possam pensar de mim. } \\
\text { Questão 07. Não estava preocupado com o que os outros pensam sobre mim. } \\
\text { Questão 11. Eu não temo o julgamento dos outros. }\end{array}$ \\
\hline $\begin{array}{l}\text { Concentração } \\
\text { imersão na tarefa }\end{array}$ & $\begin{array}{l}\text { Questão 04. Eu tenho a sensação de viver um momento de emoção. } \\
\text { Questão 08. Esta atividade me proporcionou bem-estar. } \\
\text { Questão 12. Quando eu falo sobre esta atividade, sinto uma emoção forte e } \\
\text { quero compartilhá-lo. }\end{array}$
\end{tabular}

\section{3. $\mathrm{O}$ jogo digital educacional SUSCITY}

O SUSCITY ${ }^{1}$ começou a ser idealizado em 2014, tendo sido desenvolvido pela equipe do NAPEAD (vinculado à Secretaria de Educação à Distância da UFRGS), durante o ano de 2015/2016. Ele está disponível na loja PlayStore (Android) e no repositório LUME (UFRGS), onde se pode fazer o download do projeto Unity, incluindo o código fonte e as artes. O objetivo do jogo é estimular o debate sobre a gestão dos recursos do SUS (Sistema Único de Saúde). Idealizado para complementar as atividades presenciais da disciplina de Saúde Coletiva e Bioética, o jogo simula uma cidade que precisa ser administrada pelo jogador.

Os princípios operacionais de SUSCITY referem-se à administração das cinco opções de unidades de saúde (Figura 1A): Unidade Básica de Saúde (UBS), Farmácia Popular, Unidade de Pronto Atendimento (UPA), Hospital e Emergência, administrados em função das características das doenças da população, das verbas disponíveis à gestão e considerando os indicadores de gravidade da doença e as condições de saúde dos habitantes. As metas (Figura 1B), que versam o número mínimo de curas e a porcentagem máxima de óbitos, o número de doentes da cidade, o perfil da doença e o orçamento do fundo reservado à saúde, mudam em cada fase, ficando cada vez mais difíceis de ser alcançadas (Perry et al, 2016). Cada fase tem um tempo de 3 minutos, podendo ser acelerado pelo jogador. O jogador deve implantar Unidades de Saúde que contemplem as necessidades de uma população $100 \%$ doente, em tempo hábil para que sejam curados, tentando não deixar dívidas para a próxima gestão. Em termos de design de jogos, SUSCITY é um jogo em que não se pode vencer - segundo Ruggiero e Becker (2015) este tipo de jogo tem maior potencial para promover questionamentos e estimular o desafio. Em SUSCITY, por mais que o jogador avance, no final ele está sempre muito endividado e não consegue atingir as metas da fase. Até hoje, ninguém passou da $9^{\circ}$ fase do jogo.

\footnotetext{
${ }^{1}$ https://lume-re-demonstracao.ufrgs.br/suscity-ii/index.html

V. $15 \mathrm{~N}^{\mathrm{o}} 1$, julho, 2017
} 

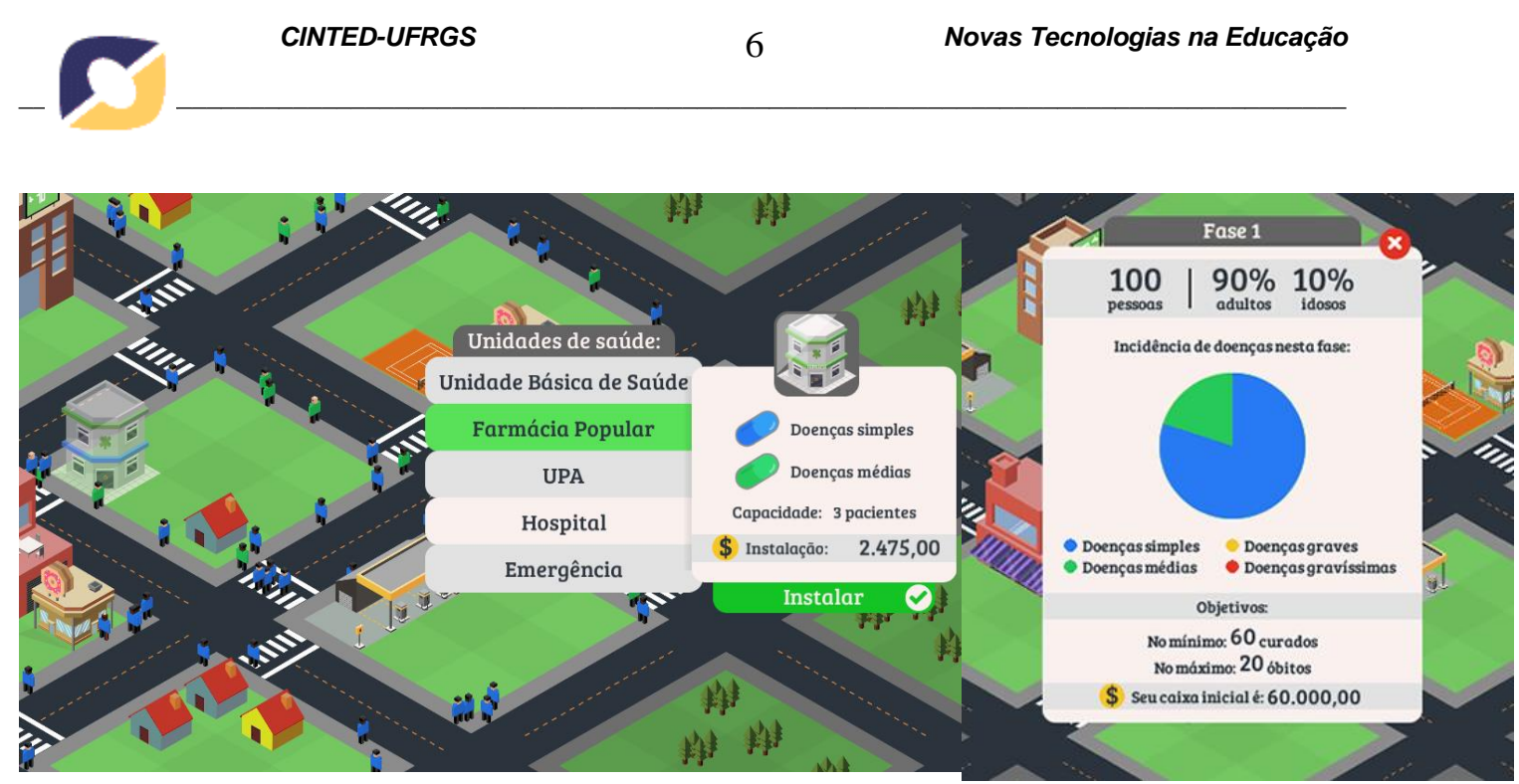

Figura 1. Opções de Unidades de Saúde e apresentação das metas do jogo.

\section{Protocolo metodológico}

O SUSCITY foi avaliado por duas turmas de alunos do curso de Graduação em Farmácia, em sessões de aproximadamente 1 hora e 40 minutos, na terceira semana letiva do semestre 2016/02. A idade dos alunos variou entre 18 e 23 anos, com exceção de uma das turmas que apresentou dois estudantes com idades de 45 e 60 anos. Trinta e dois alunos eram mulheres e 14 eram homens. Os alunos já conheciam o jogo, pois a professora o havia apresentado na aula anterior. Contudo, antes de iniciar a sessão, apresentou-se novamente o jogo, descrevendo os objetivos, mecânicas, elementos de interface e demais atributos. Além disso, esclarecemos que o objetivo da pesquisa era verificar problemas na compreensão dos elementos do jogo, bem como o nível de satisfação dos jogadores quanto a aspectos como estética e jogabilidade de forma geral, e não testar seus conhecimentos sobre a disciplina. Também ressaltamos que a participação na pesquisa era voluntária, e distribuímos um Termo de Consentimento de Participação na Pesquisa, que continha os dados de contato dos pesquisadores, descrevia os objetivos e procedimentos de coleta e armazenamento de dados da pesquisa. Cada estudante recebeu duas cópias do Termo, assinadas pelas autoras deste artigo, e guardou uma delas consigo.

A url do jogo e dos questionários ${ }^{2}$ estavam no Moodle da disciplina, de forma que os estudantes conseguiam facilmente acessar o jogo. Eles jogaram por cerca de 40 minutos, e à medida que percebiam ter condições de responder o questionário sobre o jogo, acessavam o link. Alguns alunos tiveram dúvidas sobre recursos específicos do jogo (reiniciar, passar o tutorial e acelerar o tempo). Não houve dúvidas em relação ao preenchimento do questionário.

O questionário tinha duas partes: aspectos objetivos de usabilidade (elaboradas pelas autoras) e aspectos afetivos (usando o questionário Eduflow). Os questionários estavam separados em páginas diferentes, de forma que as questões não estavam misturadas. Todas as questões eram respondidas por meio de um escala de 7 pontos, com âncoras nas extremidades, nomeadas "Discordo plenamente" (esquerda) e "Concordo plenamente" (direita). As perguntas eram invertidas, ou seja, as respostas consideradas positivas ora estavam no extremo esquerdo, ora no extremo direito da escala. Havia, no final, uma questão que pedia que o jogador escrevesse sua avaliação do jogo.

\footnotetext{
${ }^{2}$ https://goo.gl/forms/liskqo6ToV3XdjbS2
}

V. $15 \mathrm{~N}^{\mathrm{o}} 1$, julho, 2017 


\section{Resultados}

Na Tabela 1 pode-se ver que as médias em relação aos aspectos objetivos de usabilidade foram altas, o que indica que os elementos de interface e jogabilidade estavam claros. A média para a questão "entendi como se joga" foi 82,6 e para a questão "entendi porque atingi o objetivo de cada fase" foi 83. Ressalta-se que a mecânica do jogo havia sido explicada na aula anterior, inclusive com uma demonstração antes do início da sessão de avaliação. Outros bons resultados referem-se à qualidade visual do jogo ("achei bonito"; 84,5). Os resultados considerados ruins - pois desejava-se uma média maior - foram para as questões "achei o jogo chato" $(88,5)$ e "apenas jogaria se fosse obrigatório" (81). Contudo, apesar das médias serem baixas. elas estão em acordo.

Todavia, algumas médias pareceram inconsistentes com respostas anteriores. A questão "os objetivos pedagógicos do jogo não ficaram claros", que, considerando a pontuação das demais questões, deveria ter tido uma média baixa, obteve média 76,5. O outro caso de incongruência é o da questão "a relação entre a cor da roupa dos habitantes, a cor do gráfico de prevalência de doenças (objetivos) e das unidades de saúde não ficou clara", que deveria ter sido alta (considerando a média das outras questões), porém foi 30. Por este motivo, realizamos uma análise de consistência interna (usando o alfa de Cronbach) do questionário, que avalia se as perguntas medem o mesma construto (compreensão do jogo). O resultado foi muito baixo: 0.44 indicando que o questionário deveria passar por uma revisão. Sendo assim, não se pode usar os resultados deste questionário para concluir sobre a avaliação de usabilidade do jogo.

Tabela 1 - resultados das questões sobre aspectos objetivos de usabilidade

\begin{tabular}{l|c|c} 
& $\begin{array}{c}\text { Média } \\
\text { (normalizada) }\end{array}$ & Desvio padrão \\
\hline Achei o jogo bonito. & 84.5 & 1.5 \\
\hline Eu apenas jogaria se fosse obrigatório. & 80.7 & 1.9 \\
\hline Entendi como se joga. & 82.6 & 1.3 \\
\hline $\begin{array}{l}\text { Os objetivos pedagógicos do jogo não } \\
\text { ficaram claros. }\end{array}$ & 76.4 & 1.4 \\
\hline $\begin{array}{l}\text { Eu entendia porque havia } \\
\text { atingido (ou não) o objetivo de cada } \\
\text { fase }\end{array}$ & 82.9 & 1.3 \\
\hline $\begin{array}{l}\text { Achei o jogo chato } \\
\text { A relação entre a cor da roupa dos } \\
\text { habitantes, a cor do gráfico de } \\
\text { prevalência de doenças (objetivos) e } \\
\text { das unidades de saúde não ficou clara. }\end{array}$ & 88.5 & 1.6
\end{tabular}

A Tabela 2 mostra as médias normalizadas dos resultados do EduFlow.

Tabela 2 - resultados das questões sobre fluxo(EduFlow).

\begin{tabular}{l|c|c} 
& $\begin{array}{c}\text { Média } \\
\text { (normalizada) }\end{array}$ & $\begin{array}{c}\text { Desvio } \\
\text { padrão }\end{array}$ \\
\hline Me senti capaz de atender às exigências da situação. & 64 & 1.44 \\
\hline Não notei a passagem do tempo & 67 & 1.98 \\
\hline Não me preocupo com o que os outros possam pensar & 69 & 1.94
\end{tabular}


de mim.

\begin{tabular}{l|c|c}
\hline Eu tenho a sensação de viver um momento de emoção. & 63 & 1.79 \\
\hline Eu sinto que o que eu faço está sob meu controle. & 61 & 1.43 \\
\hline Eu sinto que o tempo está voando muito rápido & 70 & 1.84 \\
\hline $\begin{array}{l}\text { Não estava preocupado com o que os outros pensam } \\
\text { sobre mim. }\end{array}$ & 70.6 & 1.72 \\
\hline Esta atividade me proporcionou bem-estar & 65.8 & 1.68 \\
\hline Sei o que tenho que fazer em cada etapa da tarefa. & 64 & 1.7 \\
\hline $\begin{array}{l}\text { O tempo parece fluir através de uma forma diferente do } \\
\text { que nunca. }\end{array}$ & 65.5 & 1.84 \\
\hline Eu não temo o julgamento dos outros. & 65 & 2.11 \\
\hline $\begin{array}{l}\text { Quando eu falo sobre esta atividade, sinto uma emoção } \\
\text { forte e quero compartilhá-lo }\end{array}$ & 57.9 & 1.83
\end{tabular}

Os resultados das médias para as questões sobre fluxo não foram tão altas - o que é consistente com os resultados negativos a respeito do jogo ser "chato" e ser jogado apenas se "não for obrigatório". O resultado da análise de consistência interna (novamente usando o alfa de Cronbach) do questionário de fluxo foi alto (0.86), o que era esperado, pois este instrumento já havia passado por um estudo de validação das questões quando foi publicado em 2014.

As respostas à questão apresentada no final dos questionários ("o que você achou do jogo? Pode elogiar, pode esculachar. Isso nos ajuda a melhorar.") teve muitas respostas positivas e elogios. Dentre estas, listamos:

- Gostei do jogo, com ele podemos ter uma visão da dificuldade de atingir todas as demandas com certo valor.

- É interessante, mas acho que deveria ser aplicado no ensino fundamental, como uma forma de as pessoas entenderem desde a infancia [sic] sobre os niveis [sic] de atenção a saude [sic].

- Gostei bastante da dinâmica, só achei a fase 5 super difícil de passa-la [sic].

- Gostei do jogo, achei ele importante para entendermos para que cara nível de doença e para qual unidade a pessoa deve se encaminhar, depois do jogo, ficou mais claro pra mim.

- Achei interessante e didático.

- Eu amei o jogo e vou expor para outras pessoas de fora da UFRGS. Muito interessante ver como é difícil gerir uma

Entre as questões com respostas que denotam uma avaliação ruim listamos:

- Achei o jogo interessante, porém não é algo de meu interesse pessoal.

- Achei muito tri, porém tranca demais fazendo que eu recomeçasse o jogo todas vez que isso acontecia.

- Deveria explicar melhor os comandos.

- Achei complicado

- Gostei do jogo. Mas achei dificuldade para jogar.

- Enjoativo, chega numa certa fase que se tenta de todas as maneiras e não se consegue passar de fase, mesmo.

- Achei bem criativo e bem dinâmico da [sic] pra melhorar um pouco os gráficos, fazer mais real .

Estas avaliações qualitativas não estão de acordo com o resultado dos questionários sobre aspectos objetivos, o que reforça a conclusão que esses dados não têm 
confiabilidade, e portanto, não podem ser usados para concluir sobre os aspectos de usabilidade do SUSCITY.

\section{Conclusão}

O SUSCITY é um jogo educacional sobre gerenciamento de recursos do SUS, e foi avaliado em condição experimental, com 46 estudantes do curso de Farmácia. Foram aplicados dois questionários, com a intenção de avaliar aspectos de usabilidade (objetivos) e de experiência do usuário (fluxo). Escolheu-se avaliar estes aspectos pois considera-se importante que a mecânica, os elementos da história e da interface sejam compreendidos e porque há relação entre desempenho na aprendizagem e fluxo.

Sendo assim, desenvolvemos um questionário que avaliava aspectos da mecânica e da compreensão do jogo. Poderíamos ter usado um instrumento consolidado, como heurísticas de avaliação de jogos (por exemplo: PLAY e HEP, citadas na revisão deste artigo), porém preferimos criar questões que enfocassem diretamente o jogo. Contudo, a avaliação de consistência interna (alfa de Cronbach) indicou que as questões não estavam medindo o mesmo construto - provavelmente isso seja um reflexo da forma como redigimos as questões, com as polaridades (positiva e negativa) invertidas. Se os estudantes não leram as questões com atenção, o padrão de marcação de respostas não faria sentido - e a análise das médias que questões que avaliam itens similares mostrou precisamente isso. $O$ resultado de 0.44 para o alfa de Cronbach sugere de forma muito importante que estes dados não devem ser utilizados.

Por outro lado, os resultados do Eduflow possuem alta confiabilidade (alfa de Cronbach de 0.86). Contudo, as questões não possuem polaridades invertidas (o que é uma boa prática para a redação que questões). Ainda que as respostas do EduFlow tenham consistência interna, a média foi baixa, indicando que os estudantes não estavam em fluxo. As respostas dissertativas sobre a avaliação do jogo foram positivas.

\section{REFERÊNCIAS}

Bevan, N., 2009. What is the difference between the purpose of usability and user experience evaluation methods? Workshop on User Experience Evaluation Methods in Product Development during Interact09 Conference.

O’Neil, H. F., Wainess, R.; Baker, E. L. (2005). Classification of learning outcomes: evidence from the computer games literature. The Curriculum Journal, 16(4).

Linehan, C., Kirman, B., Lawson, S.; Chan, G. (2011). Practical, appropriate, empirically-validated guidelines for designing educational games. In Proceedings of the 2011 annual conference on Human factors in computing systems - CHI '11 (p. 1979). New York, New York, USA: ACM Press

Brom, C., Preuss, M.; Klement, D. (2011). Are educational computer micro-games engaging and effective for knowledge acquisition at high-schools? A quasi-experimental study. Computers and Education, 57(3), 1971-1988.

Gee, J. P. (2008). Cats and Portals: Video Games, Learning, and Play. American Journal of Play, 1(2), 229-245.

Gee, J. P .; Morgridge. (2007). T. Being a Lion and Being a Soldier Learning and Games. In. Facer (Ed.), Savannah: Mobile Gaming and Learning? (pp. 1027-1041).

Gee, J. P. (2009). Deep Learning Properties of Good Video Games: How Far Can They Go? In Serious Games: Mechanisms and Effects (pp. 67-82). New York: Routledge.

Clark, R. E., Feldon, D. F. (2005). Five common but questionable principles of multimedia learning. Cambridge Handbook of Multimedia Learning, (2003), 1-22

Kozma, R. B. (1994). Will media influence learning? Reframing the debate. Educational Technology Research and Development, 42(2), 7-19.

Clark, R. E., \& Feldon, D. F. (2005). Five common but questionable principles of multimedia learning. Cambridge Handbook of Multimedia Learning, (2003), 1-22. 
Li, M.-C., \& Tsai, C.-C. (2013). Game-Based Learning in Science Education: A Review of Relevant Research. Journal of Science Education and Technology.

Boehner, K. et al., 2007. How emotion is made and measured. International Journal of Human Computer Studies, 65(4), p.275-291.

Chen, L. X., \& Sun, C. T. (2016). Self-regulation influence on game play flow state. Computers in Human Behavior, 54, 341-350.

Desurvire H., Wiberg C. (2009) Game Usability Heuristics (PLAY) for Evaluating and Designing Better Games: The Next Iteration. In: Ozok A.A., Zaphiris P. (eds) Online Communities and Social Computing. OCSC 2009. Lecture Notes in Computer Science, vol 5621. Springer, Berlin, Heidelberg.

Desurvire, H., Wiberg, C. (2009). Game usability heuristics (PLAY) for evaluating and designing better games: The next iteration. In Lecture Notes in Computer Science (including subseries Lecture Notes in Artificial Intelligence and Lecture Notes in Bioinformatics) (Vol. 5621 LNCS, pp. 557-566).

Desurvire, H., Wiberg, C.: Master of the Game: Assessing Approachability in Future Game Design. In: ACM CHI 2008 conference, CHI 2008 International Conference on Computer Human interaction Florence Italy (2008)

González Sánchez, J.L., Gutiérrez Vela, F.L., 2014. Assessing the player interaction experiences based on playability. Entertainment Computing 5, 259-267.

Gonzalez, J. L. G, Vela, F.L., 2014. Assessing the player interaction experiences based on playability. Entertainment Computing 5, 259-267.

Hamari, J., Shernoff, D. J., Rowe, E., Coller, B., Asbell-Clarke, J., \& Edwards, T. (2016). Challenging games help students learn: An empirical study on engagement, flow and immersion in game-based learning. Computers in Human Behavior, 54, 170-179.

Hassenzahl, M. et al., 2000. Hedonic and ergonomic quality aspects determine a software's appeal. Proceedings of the SIGCHI conference on Human factors in computing systems CHI 00, 2(1), p.201-208

Kiili, K., Lainema, T., de Freitas, S., \& Arnab, S. (2014). Flow framework for analyzing the quality of educational games. Entertainment Computing, 5(4), 367-377.

Malone, T. W. (1982). Heuristics for designing enjoyable user interfaces. In Proceedings of the 1982 conference on Human factors in computing systems - CHI '82 (pp. 6368). New York, New York, USA: ACM Press.

Pinelle, D.A; Wong, N.b; Stach, T.C. (2008). Heuristic evaluation for games: Usability principles for video game design. 26th Annual CHI Conference on Human Factors in Computing Systems, CHI 2008; Florence; Italy

Quigley, K. S.; Lindquist, K. A.; Barrett, L. F. 2014. Inducing and Measuring Emotion and Affect. In Handbook of Research Methods in Social and Personality Psychology. Ed(s) Reis, H. T.; Judd, C. M. Cambridge University Press. p. 220-252

Ruggiero, D.; Becker, K. (2015). Games You Can't Win. The Computer Games Journal, 4(3-4), 169-186.

Stanton, N.\& Young, M. 1999. Guide to methodology in ergonomics: Designing for human use, Taylor \& Francis.

Heutte, J., Fenouillet, F., Boniwell, I., Martin-Krumm, C., \& Csikszentmihalyi, M. (2014). Optimal learning experience in digital environments: Theoretical concepts, measure and modelisation. Symposium "Digital Learning in 21 st Century Universities". Georgia Institute of Technology (Georgia Tech), Atlanta, GA. 